\title{
Oxigenación por membrana extracorpórea y plasmaféresis en un paciente con hemorragia pulmonar grave secundaria a poliarteritis nodosa

\author{
Extracorporeal membrane oxygenation and plasmapheresis in the treatment of \\ severe pulmonary hemorrhage secondary to nodose polyarteritis
}

Dr. Rodolfo Keller ${ }^{a}$, Dr. Silvio Torres ${ }^{a}$, Dr. Thomas Iölster ${ }^{a}$, Dr. Christian Kreutzer ${ }^{a}$, Dr. Rafael Fraire ${ }^{a}$, Dra. Magdalena Grassi ${ }^{a}$, Dr. Damián Arrese ${ }^{a}$ Dr. Omar Trabadelo y Dr. Manuel Rocca Rivarola ${ }^{a}$

\begin{abstract}
RESUMEN
Se comunica el caso de un paciente de 23 meses de edad, con hemorragia pulmonar, hipoxemia refractaria, hipertensión arterial y falla renal secundarias a poliarteritis nodosa $(\mathrm{PN})$ que ha requerido asistencia simultánea con soporte cardiopulmonar extracorpóreo (ECMO), plasmaféresis, hemodiafiltración y surfactante exógeno. La PN es una vasculitis autoinmunitaria que afecta los vasos arteriales de pequeño y mediano calibres. La hipoxemia, refractaria al tratamiento con ventilación mecánica convencional y de alta frecuencia oscilatoria, fue sostenida con ECMO mientras se controló la enfermedad autoinmunitaria mediante plasmaféresis y tratamiento inmunosupresor. La combinación de ECMO con plasmaféresis es infrecuente, pero ha sido descripta en relación al trasplante de órganos, intoxicaciones, fallo cardíaco y sepsis.

Existen dos casos descriptos en la bibliografía sobre el uso combinado de estas terapéuticas para el tratamiento de hemorragia pulmonar secundaria a vasculitis autoinmunitaria en niños. Palabras clave: ECMO, plasmaféresis, poliarteritis nodosa, vasculitis autoinmunitaria, hemorragia pulmonar.
\end{abstract}

\section{SUMMARY}

We report to simultaneous use of extracorporeal life support (ECLS), plasmapheresis, hemodiafiltration and exogenous surfactant for the treatment of pulmonary hemorrhage, arterial hypertension, and renal failure secondary to nodose polyarteritis (NPA) in a 23-month-old patient. NPA is an autoimmune disease that affects small -and medium- caliber muscular arteries. Hypoxemia refractory to treatment with conventional and high frequency oscillatory ventilation was supported by ECMO while awaiting control of the autoimmune disease through the use of plasmapheresis and immunosuppressive treatment. Although the combination of ECLS with plasmapheresis is rare, it has been described during the management of cases of organ transplant, intoxication, cardiac failure, and sepsis. There are only two previous reports describing the use of this combined therapy for the treatment of pulmonary hemorrhage secondary to autoimmune vasculitis in childhood.

Key words: ECMO, plasmapheresis, nodose polyarteritis, autoimmune vasculitis, pulmonary hemorrhage.

http:/ /dx.doi.org/10.5546/aap.2012.e80

a. Hospital Universitario Austral. Pilar.

Correspondencia:

Dr. Rodolfo Keller: rkeller@cas.austral.edu.ar

Conflicto de intereses: Ninguno que declarar.

Recibido: 1-11-2011

Aceptado: 18-5-2012

\section{INTRODUCCIÓN}

La Poliarteritis Nodosa (PN) es un síndrome vasculítico con afectación de arterias de pequeño y mediano calibres, especialmente de piel, riñón, sistema nervioso periférico, músculos y tracto gastrointestinal; el compromiso de otros órganos, como pulmón, cerebro y corazón son raros. Los pacientes pueden presentar insuficiencia renal aguda (IRA) e hipertensión arterial (HTA) grave. En caso de hemorragia pulmonar, puede ser masiva y es común la necesidad de asistencia respiratoria mecánica y de tratamiento inmunosupresor. ${ }^{1}$ Se presenta el caso de un niño que requirió tratamiento con oxigenación por membrana extracorpórea (ECMO), plasmaféresis y reemplazo renal continuo.

La combinación de ECMO con plasmaféresis es poco común, pero se ha descripto en relación al trasplante de órganos, ${ }^{2-5}$ intoxicaciones, ${ }^{6,7}$ fallo cardíaco ${ }^{8,9}$ y sepsis. ${ }^{10-12}$ En una búsqueda en Pubmed, sólo dos informes comunican el empleo simultáneo de ambas terapéuticas como sostén y tratamiento de hemorragia pulmonar secundaria a vasculitis autoinmunitaria en niños. ${ }^{1,13}$

\section{Caso clínico}

Paciente de 23 meses de edad, sin antecedentes patológicos relevantes, que tras 10 días de padecer un cuadro catarral en vía aérea superior, febril e inapetente, debe ser hospitalizado por insuficiencia respiratoria grave progresiva que deviene en dos episodios de paro cardiorrespiratorio en el hospital de origen, donde se diagnostica hipertensión arterial (HTA) aunada a miocardiopatía hipertrófica e insuficiencia renal. Tras 21 días de internación es trasladado a nuestro hospital en asistencia respiratoria mecánica (ARM), con saturación $86 \%$ y TA: 200/90 mmHg, con infusión continua de nitroprusiato que debió suspenderse por signos de toxicidad.

Laboratorio relevante al ingreso:

GB 9970/ml; Hto 28\%; Hb 9,5 g/L; Tp 88 seg; 
Kptt 37 seg; EAB 7,50/21/260/16,3/-5/91\%; ácido úrico $8,3 \mathrm{mg} / \mathrm{dl}$; urea $41 \mathrm{mg} / \mathrm{dl}$; creatinina 0,6 $\mathrm{mg} / \mathrm{dl}$; glucemia: $90 \mathrm{mg} / \mathrm{dl} ; \mathrm{P} 3,2 \mathrm{mg} / \mathrm{dl} ; \mathrm{Mg}$ 1,7 meq/L; Na 130; K:3,1; Cl 104 meq/L; Ca iónico: 1,15 mmol/L; BT 0,5 mg/dl; TGO: $22 \mathrm{UI} / \mathrm{L}$; TGP: $9 \mathrm{UI} / \mathrm{L}$; colesterol $220 \mathrm{mg} / \mathrm{dl}$; proteínas totales: 4,8 $\mathrm{g} / \mathrm{L}$; albúmina: $3 \mathrm{~g} / \mathrm{L}$; metahemoglobina $<0,8 \%$.

Por interrogatorio y examen físico se descartó la presencia de nódulos cutáneos, dolores articulares o musculares.

$\mathrm{Al}$ ingreso a nuestro hospital, los registros de TA continuaban elevados y difíciles de controlar. Se administraron en las primeras $48 \mathrm{~h}$ seis fármacos antihipertensivos (milrrinona, nitroglicerina, labetalol, minoxidil, fentolamina, espironolactona) y rescates con nifedipina; posteriormente se agregó enalapril y carvedilol.

El examen ecocardiográfico reveló: dilatación de cavidades izquierdas, hipertrofia concéntrica y disfunción del ventrículo izquierdo (fracción de acortamiento de $27 \%$ ) con arterias coronarias de calibre conservado.

Presentó mejoría inicial y logró extubarse al octavo día, pero cuatro días más tarde presentó dificultad respiratoria aguda (SDRA) a causa de hemorragia pulmonar grave e hipoxemia progresiva y refractaria a ARM, tanto convencional como de alta frecuencia oscilatoria (VAFO), por lo cual ingresó de urgencia a ECMO venoarterial (V-A) (Figura 1).

FiguRA 1: Rx de tórax frente.

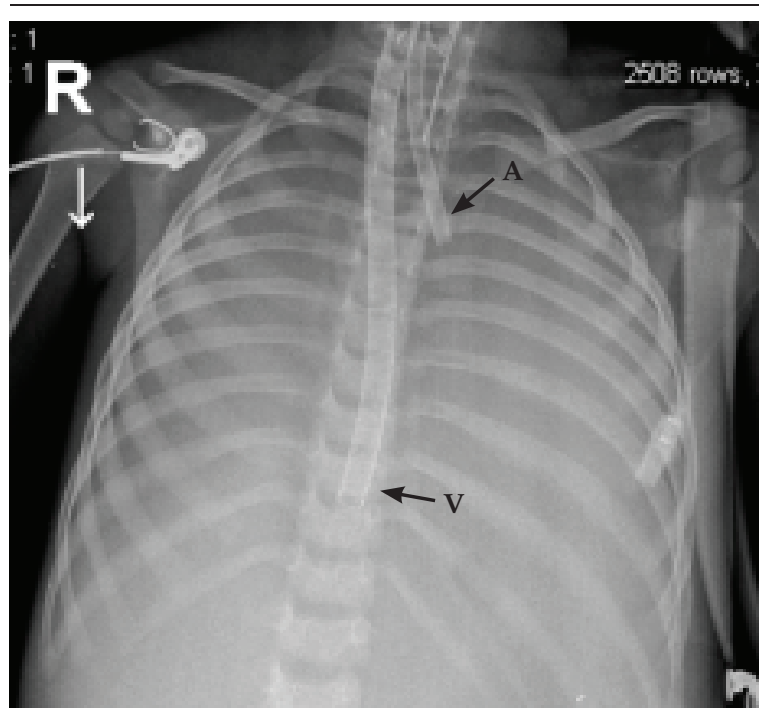

Distrés respiratorio grave. Ingreso en ECMO.

V: Cánula venosa de ECMO.

A: Cánula arterial de ECMO.
El ECMO, al reducir el flujo de sangre que atraviesa el lecho vascular pulmonar, permitió reducir el daño pulmonar y controlar la hemorragia pulmonar, a pesar de la heparinización. Se utilizó bomba centrífuga (Jostra) y oxigenador (Quarox D) a través de cánulas de ECMO arterial (14 French en carótida) y venosa (16 French en yugular) derechas. Durante el curso de ECMO se realizó una biopsia renal que descartó daño microvascular y evidenció cambios compatibles con necrosis tubular aguda.

Estabilizado el paciente, se inició tratamiento empírico con metilprednisolona, ciclofosfamida, vitamina B12 y ácido folínico. Conjuntamente, se realizaron cinco cursos de plasmaféresis o recambio plasmático terapéutico (RPT) isovolémicos de hasta 1,5 plasmemias, mediante un separador celular de flujo continuo $\left(\mathrm{Cobe}^{\circledR}\right)$. Durante el procedimiento, el paciente continuó en ECMO y con soporte de hemodiafiltración continua.

La combinación de hemodiafiltración y plasmaféresis es más simple y segura cuando se realiza sobre la línea venosa (de presión negativa) del ECMO, previo al oxigenador, que también sirve de trampa de burbujas y de recuperador de calor. Es menester un control estricto de la coagulación y de los componentes de la sangre, ya que el requerimiento de anticoagulación y de hemoderivados, pueden aumentar transitoriamente.

FIGURA 2. Rx de tórax frente, en ECMO luego del lavado broncoalveolar, surfactante y maniobras de reclutamiento.

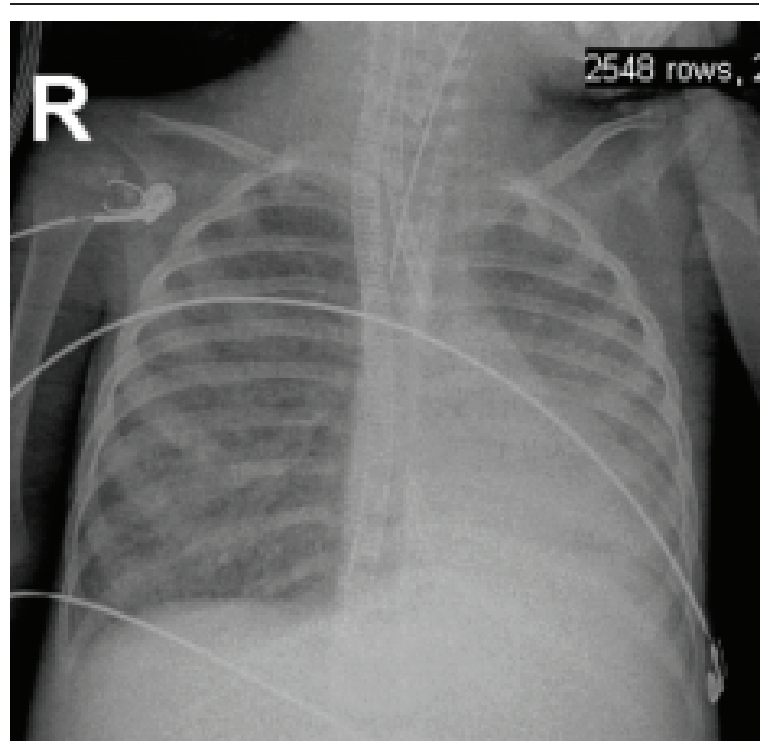


El monitoreo continuo de la saturación tisular cerebral mediante espectrometría cercana al infrarrojo (NIRS, por su sigla en inglés), resultó útil para asegurar una adecuada entrega de oxígeno tisular, demostrando una relación inversamente proporcional con el nivel de la $\mathrm{PaCO}_{2}$ y relativamente independiente de los valores de TA y de $\mathrm{PO}_{2} \cdot{ }^{19}$

Controlada la HTA y la hemorragia pulmonar, se realizó lavado broncoalveolar, seguido de la administración de surfactante pulmonar exógeno. Tras óptimo reclutamiento pulmonar en HFOV, monitorizado por radiología y gases en sangre, se logró desconectar del ECMO tras 9 días de soporte (Figura 2).

Se realizó traqueotomía 20 días más tarde de su salida de ECMO; recibió ARM hasta 10 días posteriores a la traqueotomía.

Requirió soporte renal con hemodiafiltración (19 días) y hemodiálisis (7 días). Actualmente presenta función renal aceptable, con ligero aumento de renina y aldosterona. La angiografía evidencia lesión grave de arteria renal derecha, estenosis moderada de arterias coronarias de mediano calibre (no evidentes por ecocardiografía Doppler color al inicio del cuadro) y estenosis en la vasculatura mesentérica (Figuras 3 y 4), así como ausencia de afectación de grandes vasos (arterias aorta y pulmonares).
El paciente egresó a domicilio luego de 100 días de internación, habiendo permanecido 69 días en ARM, 9 días en ECMO, 5 días en VAFO, 7 días con soporte renal continuo y tras recibir 5 sesiones de plasmaféresis. A los 9 meses de evolución no presenta signos de enfermedad pulmonar residual. Medicado con enalapril, carvedilol, losartán y amlodipina. Recibe quimioterapia con ciclofosfamida y rescates con Mesna. Fracción de acortamiento de ventrículo izquierdo: $40 \%$.

\section{Estudios realizados:}

Angiografía renal: Arteria renal derecha con doble lesión grave de polar inferior con falta de perfusión del segmento.

Arteria renal izquierda de calibre normal con segmentos completos (Figura 4).

Angiografía coronaria: Estenosis grave del segmento proximal y medio a lo largo de coronaria derecha, con lesiones de tipo excéntricos con regiones de dilatación y estenosis.

No lesión de coronaria izquierda.

Estudios Doppler de arterias humerales, radiales, cubitales, femorales comunes, superficiales y profundas, tibiales posteriores y poplíteas de calibre y velocidad de flujo conservados.

FigURA 4: Angiografía digital abdominal.

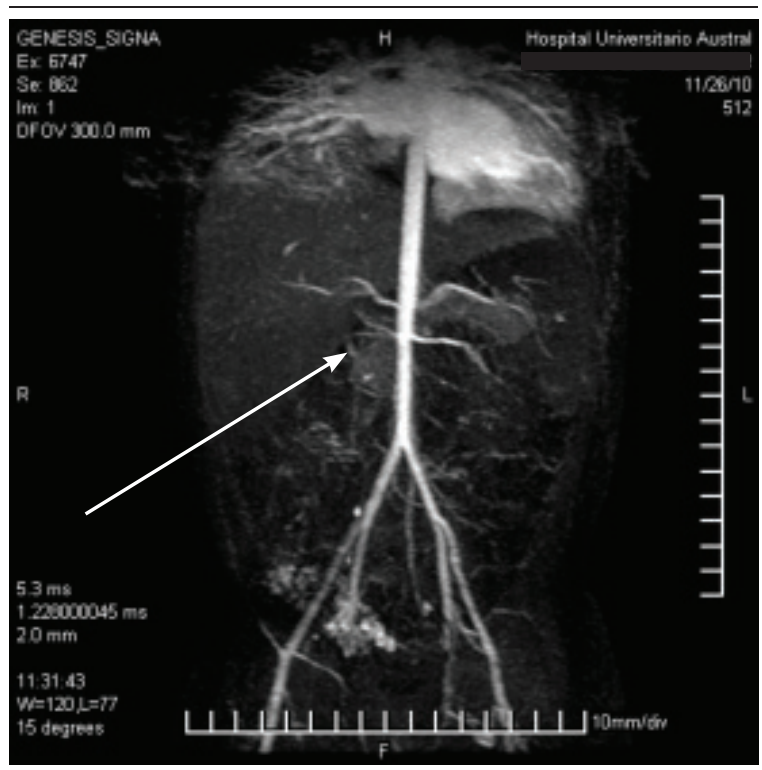

Estenosis de la arteria renal derecha.

Angiografía renal: arteria renal derecha con doble lesión grave de polar inferior y falta de perfusión del segmento. Arteria renal izquierda de calibre normal con segmentos completos.
Figura 3: Angiografía mesentérica con signos de estenosis arterial.

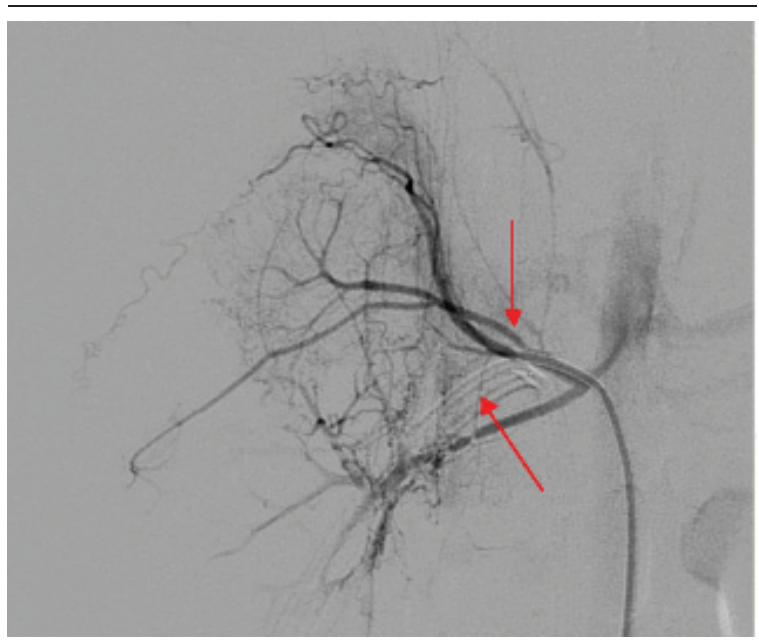


Angio-RMN abdominal: Defecto de perfusión de ambos riñones que es más evidente en el derecho e impresiona corresponder a infarto renal en evolución. Aorta y arteria renal izquierda de calibres conservados. Existen cambios de perfusión del riñón izquierdo. (Figura 4)
Angio-RMN cerebral: Sin afectación evidente.

TAC de abdomen y pelvis con contraste: Alteración en concentración y eliminación de contraste en totalidad del RD y en forma parcial del riñón izquierdo.

Ecografía de abdomen: Riñón derecho de ca-

TABLA 1. Vasculitis en la infancia: características diagnósticas y tratamiento habitual ${ }^{18}$

\begin{tabular}{|c|c|c|}
\hline Vasculitis & Características clínicas & Tratamiento \\
\hline Púrpura de Schönlein Henoch & $\begin{array}{l}\text { Púrpura palpable. } \\
\text { Dolor abdominal, artralgias o artritis, } \\
\text { compromiso renal, edema circunscripto o } \\
\text { edema de cuero cabelludo. } \\
\text { Biopsia con depósito predominante de IgA. }\end{array}$ & $\begin{array}{l}\text { Reposo. } \\
\text { Meprednisona vía oral } 1 \mathrm{mg} / \mathrm{kg} / \text { día. }\end{array}$ \\
\hline Enfermedad de Kawasaki & $\begin{array}{l}\text { Fiebre de más de } 5 \text { días de evolución. } \\
\text { Compromiso de dilatación o aneurismas } \\
\text { coronarios. } \\
\text { Descamación distal. } \\
\text { Conjuntivitis bilateral, adenopatías cervical/ } \\
\text { submaxilar, compromiso mucosa oral, } \\
\text { exantema polimorfo. }\end{array}$ & $\begin{array}{l}\text { Pulso EV } \\
\text { de gammaglobulina humana } \\
\text { a } 2 \mathrm{~g} / \mathrm{kg} / \text { dosis. } \\
\text { Aspirina a } 80 \mathrm{mg} / \mathrm{kg} / \text { día. }\end{array}$ \\
\hline Poliarteritis nudosa & $\begin{array}{l}\text { Vasculitis necrosante sistémica de pequeños y } \\
\text { medianos vasos. } \\
\text { Anormalidades angiográficas con aneurismas } \\
\text { y/o estenosis. Livedo reticularis, hipertensión } \\
\text { arterial, dolor testicular, pérdida de peso, } \\
\text { mononeuropatía o polineuropatía. } \\
\text { Compromiso vasculítico sistémico, SNC, renal, } \\
\text { cardiovascular, respiratorio y gastrointestinal. }\end{array}$ & $\begin{array}{l}\text { Analgésicos antiinflamatorios } \\
\text { no esteroideos. } \\
\text { Corticoides orales o parenterales. } \\
\text { Metotrexate. } \\
\text { Ciclofosfamida. }\end{array}$ \\
\hline Poliarteritis nudosa cutánea & $\begin{array}{l}\text { Exantema cutáneo no purpúrico con livedo } \\
\text { reticularis o sin él. } \\
\text { Nódulos subcutáneos dolorosos. } \\
\text { Asociada con SBHGA. } \\
\text { Mialgias; artralgias; artritis no erosivas. } \\
\text { ANCA negativos. }\end{array}$ & $\begin{array}{l}\text { Analgésicos/antiinflamatorios } \\
\text { no esteroideos. } \\
\text { Corticoides orales o parenterales. }\end{array}$ \\
\hline Poliangeítis microscópica & $\begin{array}{l}\text { Vasculitis necrosante autoinmunitaria } \\
\text { p-ANCA + o altos títulos de MPO-ANCA. } \\
\text { Glomerulonefritis necrosante. } \\
\text { Capilaritis pulmonar sin la presencia de lesiones } \\
\text { granulomatosas en tracto respiratorio. }\end{array}$ & $\begin{array}{l}\text { Dependiente del compromiso } \\
\text { sistémico. } \\
\text { Corticoides orales o sistémicos. }\end{array}$ \\
\hline Granulomatosis de Wegener & $\begin{array}{l}\text { Vasculitis sistémica granulomatosa. } \\
\text { Inflamación nasal oral o senos paranasales. } \\
\text { Infiltración granulomatosa en biopsia. } \\
\text { Compromiso renal glomerular. } \\
\text { Estenosis traqueal subglótica o endotraqueal. } \\
\text { c-ANCA + o altos títulos de PR3-ANCA. }\end{array}$ & $\begin{array}{l}\text { Corticoides orales o parenterales. } \\
\text { Ciclofosfamida oral o EV. }\end{array}$ \\
\hline Arteritis de Takayasu & $\begin{array}{l}\text { Vaculitis predominante de grandes vasos. } \\
\text { HTA. Claudicación de extremidades. } \\
\text { Tensión arterial diferencial > } 10 \mathrm{mmHg} \text {. } \\
\text { Anormalidades arteriales en angiografía } \\
\text { con oclusión arterial o estenosis. }\end{array}$ & $\begin{array}{l}\text { Tratamiento sintomático. } \\
\text { Corticoides orales. } \\
\text { Inmunosupresores. }\end{array}$ \\
\hline
\end{tabular}

IgA: inmunoglobulina A; SBHGA: Streptococcus betahemolítico grupo A; ANCA: anticuerpos anticitoplasmáticos; EV: endovenoso; SNC: sistema nervioso central; MPO: mieloperoxidasa; PR3: anticuerpos antiproteinasa 3; HTA: hipertensión arterial. 
racterísticas normales, de 78×32×27 $\mathrm{mm}$, con $\mathrm{RI}$ de mayor tamaño $(91 \times 47 \times 30)$ sin diferenciación corticomedular. Ambas arterias renales de calibre y velocidad de flujo conservados por este método. IRES RD 0,66 RI 0,69.

\section{DISCUSIÓN}

Los síndromes vasculíticos infantiles pueden presentarse con síntomas inespecíficos como fiebre, astenia, adelgazamiento, artromialgias y afección del estado general, o con síntomas focales que dependen de los órganos afectados (Tabla 1).

El fallo hipoxémico refractario con hemorragia alveolar difusa aunado a insuficiencia renal son manifestaciones graves asociadas a enfermedades inflamatorias sistémicas, como la $\mathrm{PN}^{, 21}$ vasculitis granulomatosas asociadas a anticuerpos (Ac) ANCA+ o enfermedad de Wegener, asociadas a Ac antimembrana basal glomerular, como el síndrome de Goodpasture $u$ otras entidades como el lupus o la poliangeítis microscópica. ${ }^{12}$ Otros diagnósticos diferenciales por descartar fueron los trastornos de coagulación y la trombofilia, como las microangiopatías trombóticas. ${ }^{20}$

Dada su alta mortalidad se requiere un diagnóstico y tratamiento precoces. El diagnóstico del caso se basó en los antecedentes, el examen físico, la determinación de reactantes de fase aguda, de los autoanticuerpos como ANCA y Ac Anti MBG (negativos), en los hallazgos angiográficos y en la biopsia renal asociados a la HTA.

Ante las fallas renal y respiratoria agudas, con hemorragia pulmonar masiva que requirió una rápida escalada terapéutica intensiva de rescate (ECMO y reemplazo renal continuo), se planteó el diagnóstico diferencial entre dos etiologías bien diferentes: poliangeítis microscópica y PN. Por lo que se intentó el control de la enfermedad con inmunosupresores y sesiones de plasmaféresis. La terapéutica inmunosupresora comienza habitualmente con glucocorticoides (pulsos de metilprednisolona), pero ante el compromiso grave se amplía a otros inmunosupresores, como la ciclofosfamida. ${ }^{14}$ En caso de vasculitis refractarias están indicados los pulsos de gammaglobulina humana y los bloqueantes de factor de necrosis tumoral alfa, como el infliximab, o los agentes anti-CD 20, como el rituximab. ${ }^{12,15}$ Si la terapia inmunosupresora sola es insuficiente, se indica el recambio plasmático (plasmaféresis). ${ }^{12}$ Las indicaciones actuales de plasmaféresis en pacientes con vasculitis sistémicas son: pacientes con Ac anti-MBG y ANCA asociados, pacientes con hemorragia pulmonar sin respuesta a terapias con- vencionales y compromiso grave desde el inicio. ${ }^{16}$ Una vez lograda la mejoría clínica se continúa el tratamiento con meprednisona, azatioprina o ciclofosfamida. ${ }^{17}$

Para el diagnóstico de PN se tomó en cuenta los criterios del American College of Rheumatology 17 y de la clasificación de vasculitis de la Paediatric Rheumatology International Trials Organisation ${ }^{18}$ sobre la base del resultado de la angiografía digital (Figura 4) y la adecuada respuesta al tratamiento con plasmaféresis e inmunosupresores.

Los otros dos casos similares comunicados por la bibliografía ${ }^{1,13}$ fueron adolescentes de 13 y 16 años, varones, portadores de poliangitis microscópica con SDRA secundario a hemorragia pulmonar y temprana terapéutica de rescate en ECMO. Ambos pacientes también sobrevivieron.

El pronóstico depende del compromiso inicial y de las secuelas inflamatorias de los órganos afectados. El curso de la enfermedad tiende a tener reagudizaciones, en ocasiones desencadenadas por infecciones asociadas (EBHA), por lo que el tratamiento debe mantenerse al menos por un año.

\section{BIBLIOGRAFÍA}

1. Di Maria MV, Hollister R, Kaufman J. Case report: severe microscopic polyangiitis successfully treated with extracorporealmembrane oxygenation and immunosuppression in a pediatric patient. Curr Opin Pediatr 2008;20(6):740-2.

2. Jhang J, Middlesworth W, Shaw R, Charette K, et al. Therapeutic plasma exchange performed in parallel with extra corporeal membrane oxygenation for antibody mediated rejection after heart transplantation. J Clin Apher 2007;22(6):333-8.

3. Saito S, Hatsumiya G, Fukushima N, Sakaguchi T, et al. Successful treatment of cardiogenic shock caused by humoral cardiac allograft rejection. Circ J 2009;73(5):970-3. Epub 2008 Dec.

4. Morales DL, Braud BE, Prile JF, Deyer WJ, et al. Use of mechanical circulatory support in pediatric patients with acute cardiac graft rejection. ASAIO J 2007;53(6):701-5.

5. Wang SS, Chou NK, Ko WJ, Chi NH, et al. Effect of plasmapheresis for acute humoral rejection after heart transplantation. Transplant Proc 2006;38(10):3692-4.

6. Kolcz J, Pietrzyk J, Januszewska K, Procelewska M, et al. Extracorporeal life support in severe propranolol and verapamil intoxication. J Intensive Care Med 2007;22(6):381-5.

7. Maclaren G, Butt W, Cameron P, Preovolos A, et al. Treatment of polypharmacy overdose with multimodality extracorporeal life support. Anaesth Intensive Care 2005;33(1):120-3.

8. Dornan RI. Acute postoperative biventricular failure associated with antiphospholipid antibody syndrome. $\mathrm{Br} \mathrm{J}$ Anaesth 2004;92(5):748-54. Epub 2004 Mar 5.

9. Zobel G, Dacar D, Rigler B, Bergen J, et al. Prolonged extracorporeal organ support in a 2-year-old boy after cardiac surgery. Intensive Care Med 1994;20(5):368-70.

10. Fortenberry JD, Paden ML. Extracorporeal therapies in the treatment of sepsis: experience and promise. Semin Pediatr Infect Dis 2006;17(2):72-9. 
11. Tabbutt S, Leonard M, Godinez RI, Sebert M, et al. Severe influenza B myocarditis and myositis. Pediatr Crit Care Med 2004;5(4):403-6.

12. Risso, JA, Mazzocchi O, De All J, Gnocchi CA. Síndrome pulmón-riñón. Medicina (Bs. Aires) 2009;69(6):663-73.

13. Agarwal HS, Taylor MB, Grzeszczak MJ, Lovvorn HN, Hunley TE, et al. Extra corporeal membrane oxygenation and plasmapheresis for pulmonary hemorrhage in microscopic polyangiitis. Pediatr Nephrol 2005;20(4):526-8. Epub $2005 \mathrm{Feb} 16$.

14. Sunderkötter C, de Groot K. Therapy of vasculitides and vasculopathies. Hartarzt 2008;59(5):382-93.

15. Thomas Golbanov C, Sridharan S. Novel therapies in Vasculitis S. Expert Opin Investig Gruds 2001;10(7):1279-89.

16. Colmegna I, Maldonado Cocco JA. Poliarteritis Nodosa revisited. Current Rheumatology Rep 2005;7(4):2888-96.

17. Adu D, Bacon PA. Classical Polyarteritis Nodosa, Microscopic poliarteritis and Churg-Strauss Syndrom. En: Oxford Text Book of Rheumatology, 2nd Ed. 9. Oxford: University Press; 1998.Págs.1358-5.
18. Ozen S, Pistorio A, Iusan SM, Bakkaloglu A, et al. Paediatric Rheumatology International Trials Organisation (PRINTO). EULAR/PRINTO/PRES criteria for HenochSchönlein purpura, childhood polyarteritis nodosa, childhood Wegener granulomatosis and childhood Takayasu arteritis: Ankara 2008. Part II: Final classification Criteria. Ann Rheum Dis 2010;69(5):798-806.

19. TyreeK, Tyree M, DiGeronimo R. Correlation of brain tissue oxygen tension with cerebral near-infrared spectroscopy and mixed venous oxygen saturation during extracorporeal membrane oxygenation. Perfusion 2009;24(5):325-31. Epub 2009 Nov 30.

20. Nguyen TC, Han YY. Plasma exchange therapy for thrombotic microangiopathies. Organogenesis 2011;7(1): 28-31.

21. Ozen S, Anton J, Arisoy N, Bakkaloglu A, et al Juvenile polyarteritis: results of a multicenter survey of 110 children. J Pediatr 2004;145(4):517-22. 